\title{
Impactos dos Caminhos na comunidade local de Santiago de Compostela: Re- sultados de um projeto de investigação em curso $^{1}$
}

\author{
Roberto Samartim²; Carlos Pazos-Justo ${ }^{3}$
}

Recibido: 1 de xullo de 2019 / Aceptado: 14 de marzo de 2020

Resumo. Este texto pretende ser um primeiro levantamento e síntese de resultados (não isento de autorreflexividade), do projeto de investigação "Discursos, imagens e práticas culturais sobre Santiago de Compostela como meta dos Caminhos" (FFI2012-35521), desenvolvido entre 2011 e 2017 por uma equipa de investigação multidisciplinar e interuniversitária vinculada à Rede Galabra (https://redegalabra.org/). O presente contributo seleciona a totalidade das publicações resultantes das análises representativas realizadas sobre secções do corpus geral do projeto referido, composto por sua vez por um corpus documental formado por livros, audiovisuais e sites, e por outro grupo de materiais constituído por inquéritos e entrevistas passados entre 2013 e 2015 a habitantes da cidade, comerciantes e visitantes (procedentes da Galiza, Espanha, Portugal e Brasil). O levantamento dos objetivos focados, o corpus analisado, a metodologia e ferramentas utilizadas e as principais conclusões ou hipóteses levantadas nos 21 produtos publicados no âmbito desse projeto de investigação permite concluir, entre outras questões, a validez da aplicação de métodos e procedimentos referenciados no trabalho por questionário ou no recurso ao Processamento da Linguagem Natural (PLN); o impacto da obra do escritor Paulo Coelho nas práticas de visitantes (nomeadamente brasileiros); ou, até, a centralidade da Igreja católica e da religião no entendimento tanto do Caminho como da própria cidade, juntamente com a construção de uma imagem de Santiago de Compostela e da comunidade local ligada à espiritualidade e o medievalismo.

Palabras-chave: Caminhos de Santiago; comunidade local; Santiago de Compostela; território; projetos de investigação; autorreflexividade.

\section{[es] Impactos de los Caminos en la comunidad local de Santiago de Compostela: Resultados de un proyecto de investigación en curso}

Resumen. Este texto pretende ser un primer abordaje y síntesis de resultados (no exento de autorreflexividad) del proyecto de investigación "Discursos, imágenes y prácticas culturales sobre Santiago de Compostela como meta de los Caminos" (FFI2012-35521), desarrollado entre los años 2011 y 2017 por un equipo de investigación multidisciplinario e interuniversitario vinculado a la Rede Galabra (https://redegalabra.org/). Esta contribución selecciona la totalidad de las publicaciones que resultan de los análisis representativos realizados sobre secciones del corpus general del proyecto referido, compuesto a su vez por un corpus documental formado por libros, audiovisuales y webs, y por otro grupo de materiales constituido por encuestas y entrevistas realizadas entre 2013 y 2015 a habitantes de la ciudad, comerciantes y visitantes (procedentes de Galicia, España, Portugal y Brasil). El abordaje de los objetivos propuestos, el corpus analizado, la metodología y herramientas utilizadas y las principales conclusiones o hipótesis retiradas de los 21 productos publicados en el ámbito de ese proyecto de investigación permite concluir, entre otras cosas, la validez de la aplicación de los métodos y procedimientos propios del trabajo por cuestionario o del recurso al Procesado del Lenguaje Natural (PLN); el impacto de la obra del escritor Paulo Coelho en las prácticas de visitantes (especialmente brasileños); o, incluso, la centralidad de la Iglesia católica y de la religión en la comprensión tanto del Camino como de la propia ciudad, al lado de la construcción de una imagen de Santiago de Compostela y de la comunidad local ligada a la espiritualidad y al medievalismo.

1 Este trabalho é resultado do projeto "Narrativas, usos e consumos de visitantes como aliados ou ameaças para o bem-estar da comunidade local: o caso de Santiago de Compostela” (FFI2017-88196-R), financiado por: FEDER / Ministerio de Ciencia, Innovación y Universidades - Agencia Estatal de Investigación.

2 Universidade da Corunha. Departamento de Letras. Grupo de Estudos Territoriais (GET-UdC) / Galabra (USC e UMinho).

Correio-e: roberto.samartin@udc.gal; http://orcid.org/0000-0001-8704-2235.

3 Universidade do Minho. Instituto de Letras e Ciências Humanas, Centro de Estudos Humanísticos. Grupo Galabra (UMinho).

Correio-e: carlospazos@ilch.uminho.pt; https://orcid.org/0000-0001-6172-3059. 
Palabras clave: Caminos de Santiago; comunidad local; Santiago de Compostela; territorio; proyectos de investigación; autorreflexividad.

\section{[en] Impacts of the Ways in the Local} Community of Santiago de Compostela: Results of a Research Project in Progress

\begin{abstract}
We introduce an initial assessment (and critical reflection) of all the results linked to the research project "Speeches, images and cultural practices on Santiago de Compostela as a goal of the Paths" (FFI2012-35521), developed between 2011 and 2017 by a multidisciplinary and interuniversitary research team linked to the Galabra Network (https://redegalabra.org/). The research takes as a point of departure a set of publications resulting from representative analyzes carried out on sections of the general corpus of the project. This corpus is composed by a documentary archive of books, audiovisuals and websites, plus surveys and interviews to inhabitants, shopkeepers and visitors (from Galicia, Portugal, Spain and Brazil) between 2013 and 2015. The survey carried out on our first production on the project allows to conclude, among other issues, the validity of the methods and procedures of work by questionnaire or Natural Language Processing (NLP); the impact of Paulo Coelho on the practices of visitors (namely Brazilians); or even the centrality of the Catholic Church and religion in the comprehension of the Way and the city, along with the construction of an image of the city / community linked to spirituality and medievalism.
\end{abstract}

Keywords: Ways of Santiago; Local Community; Santiago de Compostela; Territory; Research Projects; Critical Reflection.

Sumário. 1. Introdução. 2. O Projeto de investigação "Discursos, imagens e práticas culturais sobre Santiago de Compostela como meta dos Caminhos". 3. Discussão. 4. Síntese conclusiva. 5. Referências bibliográficas.

Referência normalizada: Samartim, R.; Pazos-Justo, C. (2020): "Impactos dos Caminhos na comunidade local de Santiago de Compostela. Apresentação de resultados de um projeto de investigação em curso", en Madrygal. Revista de Estudios Gallegos 23 Núm. Especial, pp. 307-320.

\section{Introdução}

Este contributo tenciona sintetizar e discutir as principais conclusões presentes nos resultados do projeto de investigação "Discursos, imagens e práticas culturais sobre Santiago de Compostela como meta dos Caminhos de Santiago", financiado polo Ministerio de Economía y Competitividad do governo da Espanha entre 2012 e 2015. Os trabalhos deste projeto foram desenvolvidos entre 2011 e 2017 por uma equipa internacional referenciada na Rede Galabra e integrada nalgum momento dos trabalhos por até catorze investigadores e investigadoras pertencentes a sete áreas de conhecimento diferentes (estudos na literatura e na cultura, linguística, comunicação, antropologia, sociologia, economia e ecologia) e a sete universidades localizadas na Galiza, Estado Espanhol, Portugal, o Brasil e os EUA.

Os resultados totais deste projeto atingem as 48 referências (acessíveis em https://redegalabra.org/impactos-caminho-comunidade-localsantiago-compostela/) entre as quais é possível distinguir trabalhos académicos (três trabalhos fim de mestrado e uma tese de doutoramento), comunicações em congressos internacionais (28) e publicações (16 referências, entre artigos de revistas e capítulos de livros). Deste universo de materiais selecionamos para serem analisados no presente texto um conjunto constituído por quase metade destes produtos, já que escolhemos os 20 contributos que foram efetivamente publicados (como artigos ou capítulos de livro) e a tese de doutoramento defendida no seio do projeto e acessível no repositório da USC. Destes materiais, escolhidos em virtude de terem sido publicados e estarem acessíveis publicamente em formato analógico ou digital, extraímos e abstraímos os objetivos focados, o corpus explorado, as ferramentas teórico-metodológicas ou procedimentais utilizadas e as conclusões ou hipóteses levantadas em cada um deles. Por último, discutimos a informação extraída e sintetizamos o conhecimento gerado no referido projeto de investigação até finais de 2017, ano a partir do qual começa uma segunda fase na pesquisa sobre o mesmo objeto de estudo no quadro de um novo projeto parcialmente financiado entre 2018 e 2021 polo Ministerio de Economía y Competitividad espanhol: "Narrativas, usos e consumos de visitantes como aliados ou ameaças para o bem-estar da comunidade local: o caso de Santiago de Compostela" (FFI2017-88196-R) .

4 Uma das primeiras ações realizadas pola equipa nessa segunda fase do projeto foi a síntese e divulgação parcial de resultados do projeto inicial através da exposição "A cidade, o Camiño e Nós", localizada fisicamente no passeio central da Alameda compostelana de inícios de maio a finais de junho de 2019 e cujos paineis digitalizados estão acessíveis em https://redegalabra.org/exposicion-a-cidade-o-camino-e-nos/ [consulta: 24/06/2019]. 


\section{O Projeto de investigação "Discursos, imagens e práticas culturais sobre Santiago de Compostela como meta dos Caminhos"}

O projeto "Discursos, imagens e práticas culturais..." tem como objeto de estudo o impacto dos Caminhos de Santiago sobre a comunidade local compostelana e visa duas classes de objetivos gerais. Por um lado, a equipa pretende conhecer as ideias das pessoas que visitam Santiago de Compostela em relação com os produtos por elas conhecidos e consumidos, com as suas vivências na cidade e com as imagens da comunidade local compostelana que tinham antes, durante e depois da estadia. Para isto foi preciso analisar os consumos e as práticas culturais efetivamente realizadas por essas pessoas e contrastá-los com o imaginário trazido por essas visitantes e com a lembrança posterior da experiência na cidade. Por outro lado, o projeto foca também outra classe de objetivos virados tanto para a planificação cultural como para os desenvolvimentos metodológicos específicos. No primeiro caso, interessa melhorar a qualidade de vida, a sustentabilidade das práticas e dos processos vinculados com a afluência de visitantes e a relação destas pessoas com a cidade; no segundo caso, tenciona-se avançar na elaboração de metodologias, técnicas e ferramentas específicas de análise dos impactos da afluência de visitantes nas comunidades locais recetoras; sobre ambas questões há numerosa bibliografia específica (Conde 2009; Ateljevic, Pritchard e Morgan 2007; Colombo 2011; Squire 1994; Light 2007; Frost 2006; Xie, Osumare e Ibrahim 2007; Andereck e Nyaupane 2011; Ruiz e Hernández 2007; Church e Hanks 1990; Garcia e Gamallo 2015; Guinovart e Simões 2009; Lage, Losada e Gómez 2012; entre outros).

Por outras palavras, os objetivos do projeto passam por responder perguntas como as seguintes: Que produtos culturais consomem as pessoas que visitam Santiago de Compostela? Que discursos e práticas existem sobre o $\mathrm{Ca}$ minho (e sobre Compostela e a Galiza)? Quem emite esses discursos? Como se (re)produzem e socializam as imagens construídas sobre o Caminho ou sobre a cidade? Em que medida esses discursos, imagens e práticas impactam no turismo e na população local? Que instrumentos de análise têm de ser utilizados para nos aproximarmos do nosso objeto de estudo? e, em última instância, qual é a utilidade para a comunidade (científica ou local) de uma investigação destas características?

Aos efeitos da investigação iniciada em 2011, identificamos como estratégico o período compreendido entre os anos Jacobeus consecutivos de 2010 e 2021, motivo polo qual localizamos cronologicamente a nossa pesquisa nesta primeira fase no período 2008-2015. Delimitamos também a investigação a quatro âmbitos geo-culturais concretos -a Galiza, Espanha, Portugal e o Brasil-em função de serem estas as procedências do maior número de pessoas visitantes a Compostela nessa data a quo de 2008 de acordo com o Centro de Estudos e Investigacións Turísticas da Universidade de Santiago de Compostela [CETUR-USC], que situa o português e o brasileiro como os contingentes europeu e extraeuropeu mais numerosos nesse ano (este facto vem ao encontro, aliás, das fortalezas da equipa, procedente na sua maioria dos estudos lusófonos).

Para atingir o seu objeto de estudo, no projeto foi selecionado um corpus de dous tipos: 1) um corpus documental inventariado e catalogado numa base de dados (Catalogador) construída ao efeito (Samartim 2015) e que continha no fim do período em análise (2017) 559 livros (de ficção, guias e diários de viagem), 211 webs e 90 produtos audiovisuais produzidos desde 2008; e 2) um corpus vivo, conformado por 2.081 inquéritos passados a visitantes procedentes da Galiza, do resto do Estado Espanhol, de Portugal e do Brasil; por 922 inquéritos feitos a habitantes da cidade e por outros 410 realizados a comerciantes e pessoas que desempenham atividades económicas de vário tipo em Santiago de Compostela.

Estes inquéritos foram desenhados pola equipa e conformados, em geral, polos seguintes blocos temáticos: as pessoas visitantes foram perguntadas polo processo de decisão da viagem, polos meios de informação usados durante a visita e pola gestão e organização da viagem. Por seu lado, à população local e às comerciantes foi pedido identificarem os espaços mais significativos para a comunidade, o grau de afetividade identitária sentido em relação com o concelho, os efeitos do turismo e a presença do Caminho de Santiago em Compostela. Para além de fazermos constar as correspondentes características sócio-demográficas de cada grupo, nos inquéritos passados tanto a visitantes como a comerciantes e locais figuram também questões ligadas à sua perceção 
sobre a imagem de Compostela e da Galiza, assim como relativas aos usos realizados e aos espaços frequentados na cidade.

Os 2.081 inquéritos feitos a visitantes de março de 2013 a março de 2014 distribuem-se entre pessoas procedentes da Galiza (398), da Espanha (878), de Portugal (408) e do Brasil (396). Os 929 inquéritos passados às pessoas residentes em Santiago de Compostela foram realizados do segundo semestre de 2014 a maio de 2015, diferenciando aqui as pessoas censadas em Compostela (684) daquelas que, fazendo grande parte da sua vida diária na cidade, têm moradia nos concelhos limítrofes de Teo (104) e Ames (101). Os 410 inquéritos sobre atividade económica e comercial da cidade foram passados em abril e maio de 2015 a pessoas que detentam a propriedade ou a gestão de locais comerciais abertos ao público no termo municipal santiaguês e que vendem produtos ou fornecem serviços de vário tipo tanto a população local como a visitantes (alojamento, alimentação, vestimenta, imagem e asseio pessoal, cultura, lazer, etc.).

Algumas das pessoas que preencheram o formulário do inquérito foram selecionadas para serem entrevistadas telefónica ou presencialmente (em conversas com uma duração média de 45 minutos), sendo assim realizadas e gravadas um total de 271 entrevistas a pessoas procedentes da Galiza (56), a Espanha (100), Portugal (56) e o Brasil (59). Além disto, o projeto contou também com um corpus complementar de 34 entrevistas feitas em Porto Alegre a pessoas de Rio Grande do Sul que fizeram ou tinham previsto fazer o Caminho de Santiago; com seis grupos de conversa com população local e com uma série de inquéritos de controlo realizados em 2018 a visitantes procedentes dos quatro âmbitos geográficos apontados acima (155) e a habitantes de Compostela e comerciantes (50 para cada categoria) (veja-se a síntese da informação sobre o corpus na Imagem 1).

Este corpus foi explorado pola equipa através de um conjunto de métodos e ferramentas procedimentais de variada natureza, desde a análise multidimensional de textos, consistente em boa medida numa leitura cercana de bibliografia que permitiu a extração e abstração de ideias e categorias; até a realização de análises estatísticas descritivas e fatoriais (valendo-nos do programa informático SPSS), de

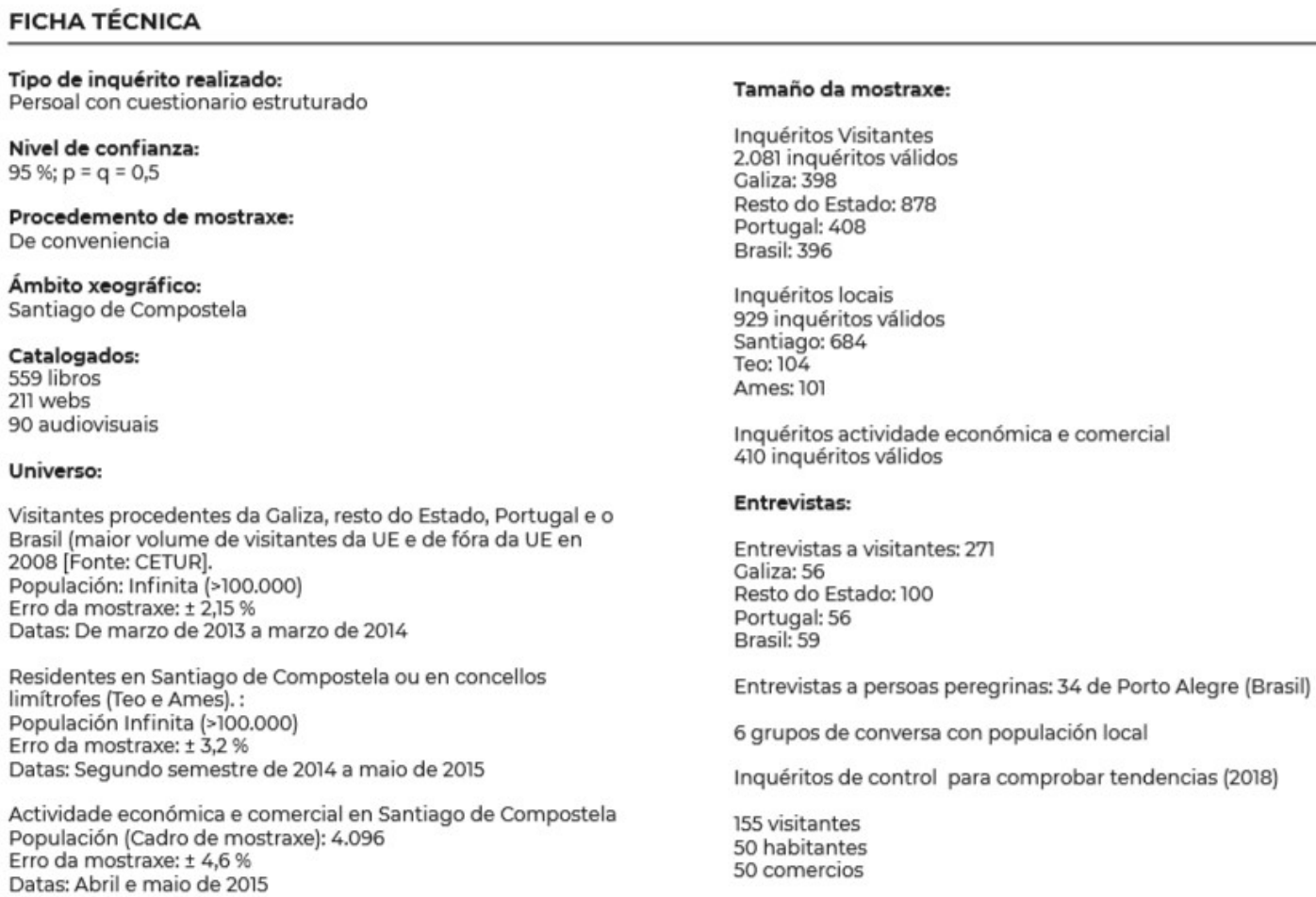

Tamaño da mostraxe:

Inquéritos Visitantes

2.081 inquéritos válidos

Galiza: 398

Resto do Estado: 878

Portugal: 408

Brasil: 396

Inquéritos locais

929 inquéritos vấlidos

Santiago: 684

Teo: 104

Ames: 101

Inquéritos actividade económica e comercial 410 inquéritos válidos

\section{Entrevistas:}

Entrevistas a visitantes: 271

Galiza: 56

Resto do Estado: 100

Portugal: 56

Brasil: 59

Entrevistas a persoas peregrinas: 34 de Porto Alegre (Brasil)

6 grupos de conversa con populación local

Inquéritos de control para comprobar tendencias (2018)

155 visitantes

50 habitantes

50 comercios 
técnicas de Processamento de Linguagem Natural $[\mathrm{PLN}]$ com recurso a vários programas informáticos (entre os quais destaca, polo sua produtividade, o Iramuteq: http://iramuteq.org/) ou da observação empírica no terreno.

Já quanto à produção do projeto, a equipa apresentou em numerosos congressos internacionais sondagens concretas sobre o corpus do projeto de investigação acima referido. Tal como é possível verificar com a consulta ao Gráfico 1, os resultados destas abordagens constituem mais de metade da produção total da pesquisa $(59 \%)$ e vários deles foram depois desenvolvidos para serem publicados em revistas ou em capítulos de livro, nutrindo assim as publicações vinculadas ao projeto, as quais supõem, por sua vez, um terço da produção total dele.

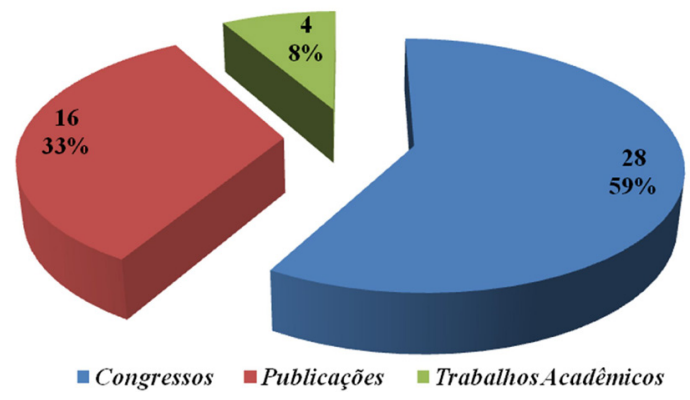

Gráfico 1. Produção geral do projeto "Discursos, imagens e práticas...". Fonte: Elaboração própria

Esta estratégia de apresentação de resultados parciais do projeto em encontros científicos internacionais, para posteriormente serem desenvolvidos em publicações (ligadas ou não com os eventos concretos), explica também o ritmo anual de publicação, concentrada fundamentalmente nos anos 2014 e (sobretudo) 2015, tal como consta mais abaixo no Gráfico 2. De facto, os anos anteriores foram dedicados a trabalhos de planificação, redação e

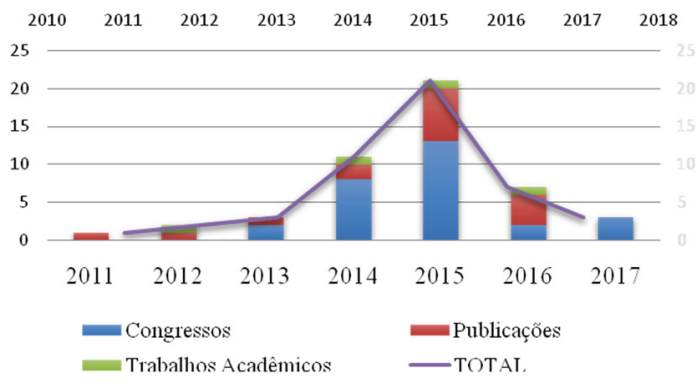

Gráfico 2: Produção anual do projeto entre 2011 e 2017. Fonte: Elaboração própria preparação da candidatura do projeto à convocatória de financiamento público em regime de concorrência competitiva lançada polo Ministerio de Economía y Competitividad do governo da Espanha no ano 2011, à conceitualização e construção da base de dados destinada à catalogação do corpus documental, ao desenho dos questionários destinados aos inquéritos a visitantes (2012), e ao preenchimento nas ruas da cidade desses inquéritos (diariamente, entre março de 2013 e março de 2014) e dos correspondentes à população local e comerciantes, num procedimento de levantamento de informação que só foi encerrado em maio de 2015. Quer a informação recolhida no Catalogador do projeto quer a levantada em várias fases através dos inquéritos passados nesse período pôde ser parcialmente processada para a participação de vários membros da equipa nesses congressos científicos internacionais.

Quanto à distribuição individual da produção do projeto em foco (Gráfico 3), ela é irregular em boa medida por causa dos diferentes momentos em que os membros da equipa se incorporam ao projeto. Neste sentido, Carreira, Carral, López Pena e del Río integram a equipa só desde 2016, altura em que já tinha concluído tanto o grosso do levantamento do corpus do projeto como o período de financiamento do Ministerio de Economía y Competitividad espanhol, e quando várias pessoas já tinham apresentado sondagens sobre esse corpus nalgum dos 21 encontros científicos em que foram divulgados resultados. Entre eles podemos destacar o XI Congresso da Associação Internacional de Lusitanistas celebrado em Cabo Verde em julho de 2014, porque é neste evento onde se concentra a maior participação de membros da equipa com contributos vinculados ao projeto, que sairão do prelo como capítulos de livro no ano seguinte (Bello Vázquez 2015b, Rodríguez Prado 2015b, Villarino 2015 e Samartim 2015).

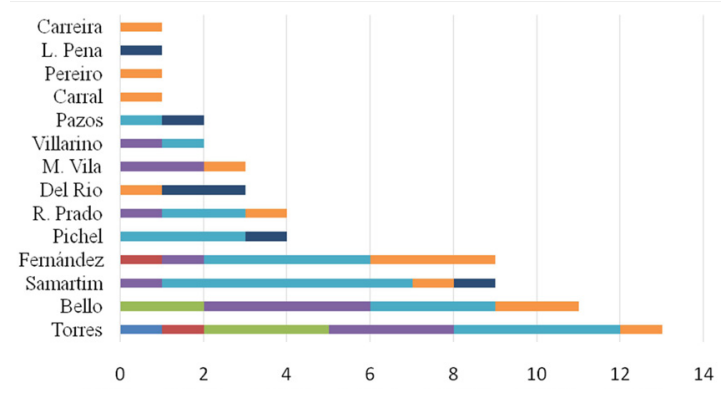

Gráfico 3: Produção individual do projeto entre 2011 e 2017. Fonte: Elaboração própria 
Por outro lado, também uma certa especialização nas tarefas vinculadas ao projeto contribui para a explicação tanto da distribuição individual dos seus resultados como dos assuntos neles focados. Assim, no conjunto das 48 referências resultantes do projeto em análise, os contributos de Pichel, Martínez Vila e Fernández Rodríguez estão relacionados com o momento em que iniciam o seu percurso investigador, com o desenvolvimento deste e com as suas obrigas académicas como pessoal em formação, sendo responsáveis por resultados vinculados aos seus Trabalhos Fim de Mestrado [TFM] (Fernández Rodríguez 2012, Martínez Vila 2014 e Pichel 2015) e à tese de doutoramento defendida no ano 2016 por Fernández Rodríguez, neste caso achegando contributos prévios tanto individuais (Fernández Rodríguez 2015) como em colaboração com algum dos seus orientadores, os professores Samartim e Torres Feijó (Fernández Rodríguez e Samartim 2015a, 2015b e 2016; Torres Feijó e Fernández Rodríguez 2014).

Igualmente, a pesar do carácter relativamente transversal dos contributos do pessoal investigador senior que acumula uma maior produção (Torres Feijó, Bello Vázquez e Samartim), a referida especialização é patente também nos assuntos focados por várias pessoas integrantes da equipa. Assim acontece na abordagem centrada na vertente audiovisual do projeto ao cuidado de López Pena (2017); nos estudos sobre o turismo sob a responsabilidade do professor Pereiro (2016); ou na focagem imagológica em que trabalha Pazos-Justo (2017), no que supõe, aliás, também a primeira achega a conceitos como reinventio e ao impacto das políticas culturais e turísticas nessa ressemantização da cidade verificada após a institucionalização polo governo autonómico galego do Ano Santo Xacobeu de 1993 (e que serão desenvolvidas no tramo final do projeto em parceria com outros membros da equipa, por exemplo em Pazos-Justo, del Río e Samartim 2018).

Também é detetável essa relativa especialização nas abordagens sobre a sustentabilidade ambiental de Carral e Carreira (que assinam com Martínez Vila e Torres Feijó em 2016 um desenvolvimento do TFM apresentado polo primeiro e orientado polo segundo na USC dous anos antes), ou nas pesquisas sobre o corpus brasileiro efetuadas tanto por Villarino
Pardo (2015) e Rodríguez Prado (2015a) como por Bello Vázquez (2015c e 2016), ainda que estas duas últimas investigadoras tenham realizado também trabalhos com base no corpus de visitantes portugueses (Bello Vázquez 2015b, Rodríguez Prado 2015b).

Da mesma maneira que a elaboração metodológica e conceitual é explorada desde os inícios do projeto principalmente polo seu investigador principal [IP], o professor Torres Feijó (2013 e 2015a), e por Bello Vázquez (2015a), a descrição e teste de ferramentas destinadas tanto à catalogação e categorização de materiais como à análise textual informatizada descansa nesta primeira fase fundamentalmente nos trabalhos individuais de Samartim (2015 e 2016) e de Fernández Rodríguez (2015, 2016a, 2016b), e nos contributos conjuntos de ambos (Fernández Rodríguez e Samartim 2015a, 2015b e 2016); na seguinte fase da investigação este aspecto será reforçado com os desenvolvimentos relativos à aplicação de técnicas de textometria ao corpus do projeto realizados por Iriarte (com Gamallo e Simões 2018).

Tal como foi apontado, dentre as 48 referências resultantes do projeto escolhemos para serem analisadas como parte do corpus do presente contributo aquelas que foram efetivamente publicadas (um conjunto de 20 textos), juntamente com a tese de doutoramento de Luisa Fernández Rodríguez (2016a), o único dos quatro trabalhos académicos realizados no âmbito da pesquisa acessível neste momento para consulta através do repositório da USC. Lembramos que destes materiais interessa-nos: 1) os objetivos focados, 2) o corpus movilizado, 3) os métodos e ferramentas utilizados e 4) as conclusões ou hipóteses retiradas de cada um dos 21 contributos que constituem o corpus do presente trabalho. Os quatro elementos referidos foram extraídos destas 21 referências concretas e sintetizados através de categorias elaboradas ao efeito (apresentamos abaixo um fragmento da tabela utilizada para este fim, a modo apenas de exemplo demonstrativo do procedimento). A informação assim retirada será analisada e discutida na continuação, levando em conta os elementos que supõem um avanço no conhecimento sobre o objeto de estudo atendido no projeto e reflexionando, também, sobre os eventuais défices ou ausências detetadas na trajetória da equipa que o sustenta durante o período em que ele vigorou. 


\begin{tabular}{|c|c|c|c|c|}
\hline $\begin{array}{c}\text { Autoria / } \\
\text { Ano }\end{array}$ & Objetivos & Corpus & $\begin{array}{l}\text { Métodos / } \\
\text { Ferramentas }\end{array}$ & Conclusões / Hipóteses \\
\hline $\begin{array}{l}\text { 1. Pazos-Justo } \\
2017\end{array}$ & $\begin{array}{l}\text { Impacto do } \\
\text { turismo }\end{array}$ & $\begin{array}{l}\text { Revisão biblio- } \\
\text { gráfica } \\
327 \text { e } 252 \text { inq. } \\
\text { visitantes }\end{array}$ & $\begin{array}{l}\text { Imagologia } \\
\text { Análise multidi- } \\
\text { mensional de textos }\end{array}$ & $\begin{array}{l}\text { 1. Funcionamento do imago- } \\
\text { tivo de afinidade: comunidade } \\
\text { galego-portuguesa } \\
\text { 2. Impacto da imagem do Jacobeu } \\
\text { (políticas públicas) } \\
\text { 3. Primeiras referências à Reinventio }\end{array}$ \\
\hline $\begin{array}{l}\text { 2. Bello } \\
\text { Vázquez } 2016\end{array}$ & $\begin{array}{l}\text { Consumos } \\
\text { culturais } \\
\text { peregrinos } \\
\text { Porto Alegre } \\
\text { (PA)? }\end{array}$ & $\begin{array}{l}34 \text { entrevistas } \\
\text { em profundidade } \\
\text { (Porto Alegre) }\end{array}$ & $\begin{array}{l}\text { Análise multi- } \\
\text { dimensional de } \\
\text { textos. } \\
\text { Análises estatísti- } \\
\text { cas descritivas }\end{array}$ & $\begin{array}{l}\text { 1. Existência duma cultura e uma ética } \\
\text { do Caminho } \\
\text { 2. "Neutralidade cultural": Experiência } \\
\text { internacional de pronto consumo } \\
\text { 3. [Endogamia/ Ocultamento]: não in- } \\
\text { teração com cultura/ população local, } \\
\text { só com outros peregrinos } \\
\text { 4. ["Distinção"]: desapego/ austeridade } \\
\text { ("jogar a ser pobre") } \\
\text { 5. ["Espiritualidade"]: viagem espiritual } \\
\text { (não turismo) }\end{array}$ \\
\hline $\begin{array}{l}\text { 3. Fernández } \\
\text { Rodríguez } \\
\text { e Samartim } \\
2016\end{array}$ & $\begin{array}{l}\text { Impacta Pau- } \\
\text { lo Coelho } \\
\text { em práticas/ } \\
\text { consumos } \\
\text { ligad@s ao } \\
\text { Caminho? }\end{array}$ & $\begin{array}{l}\text { Revisão } \\
\text { bibliográfica }\end{array}$ & $\begin{array}{l}\text { Análise multi- } \\
\text { dimensional de } \\
\text { textos. } \\
\text { Observação } \\
\text { participante }\end{array}$ & $\begin{array}{l}\text { 1. Confirma-se o impacto do discurso } \\
\text { de Paulo Coelho } \\
\text { 2. Práticas/ Consumos/Imagem da } \\
\text { cidade: espiritualidade, medievalismo } \\
\text { (ex. souvenirs templários) } \\
\text { 3. Ocultamento da cultura/ população } \\
\text { local }\end{array}$ \\
\hline
\end{tabular}

Tabela 1: Procedimento utilizado para o levantamento de informação sobre o corpus (exemplo). Fonte: Elaboração própria

\section{Discussão}

Entre os resultados efetivamente publicados, uma primeira linha de análise diz respeito ao desenho, construção e teste de ferramentas, nomeadamente da Base de Dados idealizada e construída para a recolha, catalogação, categorização e exploração do corpus documental (assunto atendido por Samartim 2015). Verifica-se que esta ferramenta, construída na sequência da trajetória tecnológica de Galabra (abordada em Samartim 2016), permite censar e catalogar grandes quantidades, variedades e origens de corpus; possibilita a aplicação de múltiplas e variadas abordagens e análises de vário tipo (quantitativas, qualitativas, relacionais, ...) e, por sua vez, também pode incorporar o recurso a outras ferramentas auxiliares
(Análise de Redes Sociais, textometria, PLN, etc. $)^{5}$. De facto, fazem parte dos objetivos focados na conceitualização desta ferramenta tanto a categorização automática como a geolocalização de conteúdos e materiais, assuntos que foram agendados para fases posteriores da investigação em função dos recursos disponíveis.

Quanto às restantes ferramentas desenvolvidas ou utilizadas no projeto, na sequência da tese de Fernández Rodríguez (2016a) foram feitas abordagens de métodos e procedimentos com suporte informático destinados ao teste de técnicas e ferramentas apropriadas para o trabalho com grandes volumes de corpus textual, através de análises não supervisionadas de materiais; estas achegas foram apresentadas em

\footnotetext{
Parte deste conhecimento sobre a aplicação de tecnologias digitais ao estudo da literatura foi compartilhado com o Grupo Leitura e Acervo Literário da Universidade de Passo Fundo (UPF). Desta colaboração resultou o desenho de uma base de dados adaptada às necessidades do acervo do escritor gaúcho Josué Guimarães, apresentada em Samartim e Rettenmaier (2015).
} 
congressos e não foram ainda publicadas (Fernández Rodríguez 2015, Fernández Rodríguez e Samartim 2015a e 2015b), mas delas resulta o uso preferente em trabalhos posteriores da equipa do programa informático Iramuteq já nomeado acima. Por seu lado, o processo de elaboração ou a apresentação dos inquéritos e das entrevistas desenhadas no âmbito da pesquisa não foi desenvolvido por enquanto em nenhum contributo das pessoas que integram a equipa.

Um segundo âmbito de análise tem a ver com as elaborações metodológicas e conceituais desenvolvidas no projeto. Neste sentido, destacam as achegas em volta dos conceitos de Sustentabilidade e Afetividade Identitárias realizadas por Torres Feijó (2013, 2015a e 2015c), para quem:

Identity sustainability is what guarantees the continuity of a community and the consensus about the community's identity, understanding sustainability in a twofold way: as the action of the community to keep the same items, and as the action of the community to preserve its identity and its limits. (Torres Feijó 2015c: 148)

Ao lado destas achegas conceituais viradas para o entendimento do objeto de estudo e das suas derivadas, alguns contributos foram dedicados a reflexionar em volta das responsabilidades e dos impactos (pessoais, sociais, ambientais) da própria atividade investigadora da equipa. Neste sentido destaca o conceito de Responsabilidade Social Investigadora [RSI] elaborado igualmente polo professor Torres Feijó:

By Socially Responsible Research we mean (SRR) the commitment to share results and the explanations of the research processes that lead to them with the human subjects involved in it and/or the social sectors potentially interested in or affected by those results. A generic definition of SRR could describe it as the commitment acquired by a research entity with the communities that are potentially interested in or affected by its research. We talk about result analysis and explanation in order to refer to those actions that lead to the understanding of research questions and their objectives, the processes used to arrive to the results and the information derived from them. (Torres Feijó 2015b)

Também atendem esse mesmo objetivo os conceitos de "Resiliência Académica" e "Sustentabilidade Investigadora" propostos por Samartim:

Envolvido neste processo de mudança, transição e auto-reflexibidade, o grupo Galabra entende a investigação como serviço público destinado ao bem comum, desenvolve práticas investigadoras viradas para a explicação (científica/ empírica) de fenómenos sociais relacionados com a cultura, e coloca no centro dos seus interesses a resiliência académica, isto é, a capacidade de adaptar a natureza dos seus discursos e das suas práticas aos estímulos externos ao campo académico, com o fim de contribuir para a sustentabilidade investigadora, a continuidade e o bem estar da(s) comunidade(s) em que se insere e atua. (Samartim 2016: 272; itálicos nossos) ${ }^{6}$

Por outro lado, os primeiros resultados vinculados ao projeto, da autoria de Torres Feijó, exploram corpus documental (Torres Feijó 2011) e ficcional (Torres Feijó 2012) e estão destinados ao levantamento de hipóteses que se verificaram centrais para posteriores desenvolvimentos e sobre as quais assenta, em boa medida, a conceitualização geral da pesquisa. Assim, num primeiro caso, as hipóteses levantadas sobre os três macrodiscursos em que descansa a visão atual da cidade e do Caminho (responsabilidade da Igreja Católica, de organizações internacionais como a UNESCO e do escritor Paulo Coelho e os seus continuadores) virão a ser acompanhadas em vários dos resultados do projeto (como veremos abaixo) e também confrontadas -e confirmadas - na tese de Luisa Fernández Rodríguez (2016a) ${ }^{7}$. O mesmo

6 O conceito de Sustentabilidade Investigadora é abordado em trabalhos posteriores por Samartim (2019), que o define como a "propriedade de pessoas, organizações ou comunidades que mantêm no tempo as práticas e processos próprios do trabalho (profissional ou não) na investigãção dita científica, sem ultrapassarem a capacidade de carga das pessoas, equipas ou comunidades envolvidas, fazendo um uso adequado (eficiente, eficaz, ...) equitativo e autogerido de capitais ou recursos (naturais, sociais, culturais, económicos, ...), tempo e tecnologias, minimizando os consumos de bens ou materiais não renováveis e contribuindo para o bem-estar e a realização humana e social tanto do pessoal investigador como das comunidades que o acolhem". Nesse mesmo contributo (Samartim 2019) a capacidade de carga é definida como o "umbral máximo de impactos negativos significativos gerados no ambiente (natural, institucional, social, ...) que pode suportar uma pessoa ou organização sem alterar as suas condições de estabilidade, coesão e continuidade".

7 Para Torres Feijó (2014: 293): “os diversos discursos enunciados correspondem a diversas estratégias, que podem ser sintetizadas, no caso das organizações [...] envolvidas, no apoio a um impulso proselitista da Igreja Católica, 
pode ser dito para a proposta lançada em 2012 por Torres Feijó em relação com a elaboração de um conjunto de parâmetros de cultura, com que o IP do projeto pretende fixar tanto as motivações dos visitantes para realizarem o caminho ou visitarem a cidade (motivação desportiva, cultural, espiritual, convivencial e de aventura) como as ideias e imagens presentes nos produtos culturais sobre o fenómeno jacobeu ou sobre a própria cidade de Compostela: património (material e imaterial), paisagem e organização do território, religião e espiritualidade, esoterismo e mistério; juntamente com os modos de vida, idiossincrasias e identidade diferencial da(s) comunidade(s), com presença aqui também da gastronomia e da língua galega (Torres Feijó 2012).

De facto, em relação ainda com os discursos, as imagens e as ideias sobre a cidade e o Caminho, múltiplos trabalhos da equipa exploram (em ocasiões de modo contrastivo e com a aplicação de técnicas de PLN) tanto o corpus documental (nomeadamente o literário) como os resultados dos inquéritos passados a visitantes -quer a pessoas procedentes de Portugal (como em Rodríguez Prado 2015b) quer a visitantes chegadas do Brasil (como em Villarino Pardo 2015 ou Bello Vázquez 2014)-, para conferir a existência desses parâmetros de cultura acima referidos. O mesmo acontece para o caso das macronarrativas, toda a vez que vários contributos (Bello Vázquez 2016; Fernández Rodríguez e Samartim 2016; Fernández Rodríguez, del Río e Rodríguez Prado 2016) exploram os impactos dos discursos de Paulo Coelho nas práticas, nos consumos e nos discursos dos visitantes (sobretudo) brasileiros.

Para além de se derivar desses mesmos resultados uma maior presença do Caminho e da peregrinação na literatura e nos discursos institucionais do que nas informações fornecidas polas pessoas visitantes, verifica-se neles a elaboração de uma determinada imagem tanto de Compostela (e da Galiza) como do Caminho (por exemplo em Torres Feijó 2014). Esta imagem descansa na identificação da cidade com um santuário cristão (com a Catedral no seu centro), constituído em meta dos vários
Caminhos que para ele conduzem; essa cidade é reduzida fundamentalmente à zona monumental de Compostela e projetada como um cenário patrimonial medievalizante, apropriado para a vivência e a realização de práticas espirituais e esotéricas. Tanto na própria cidade como nas populações atravessadas polo Caminho, as comunidades e culturas locais estão ausentes. Isto é assim em boa medida porque os Caminhos a Santiago constroem a sua própria cultura e relacionamentos, caracterizada a primeira por uma ética da austeridade e o desapego, e os segundos pola interação basicamente com outras pessoas peregrinas, envolvidas na mesma viagem iniciatica e espiritual (que não turística). A não correspondência entre produção, consumos e práticas de locais e visitantes (turistas) apontada em Carral et al (2016) reforça igualmente essa ideia de auto-referencialidade do Caminho (endogamia) e de falta de espaços e práticas de interseção entre locais e visitantes.

Ainda que esta é a caracterização geral que se deriva do corpus analisado, também é possível matizar esta construção de Compostela como imagem de destino em função da origem nacional do corpus de entrevistas a visitantes. Assim, Fernández Rodríguez, del Río e Rodríguez Prado (2016) verificam que ao lado da centralidade da Catedral e de outros elementos ligados à peregrinação e ao Caminho (estes últimos com menor peso nas informações procedentes de Portugal) presente nas três nacionalidades de informantes em foco neste contributo (espanhola, portuguesa e brasileira), a paisagem e a idiossincrasia é um dos elementos destacados principalmente por visitantes espanholas e portuguesas, sendo a gastronomia um dos parâmetros culturais mais valorizados no caso das pessoas procedentes da Espanha e do Brasil (ainda que estes em menor medida, já que referem mais maciçamente a religião e o património histórico e arquitetónico da cidade). Só as informantes portuguesas apontam para uma identificação intercomunitária com as pessoas naturais da Galiza, algo que não está presente nem nas enquisas passadas a visitantes procedentes do resto do Estado Espanhol nem no

sobretudo dirigido às pessoas jovens e no alicerçar esta Igreja como base e fundamento da Europa e na vontade de articulação e consolidação europeias pela EU; e, igualmente, na proteção e valoração do património material e imaterial que a UNESCO persegue. O discurso de Paulo Coelho [...] estaria atraído e impulsado pelos discursos das organizações citadas. De facto, não se afasta deles, pelo menos explicitamente: é um itinerário espiritual pelo Caminho de Santiago". 
contingente brasileiro (que sim destaca a língua galega como elemento identitário diferencial da comunidade local).

Por último, são também vários os trabalhos que apontam para essa perceção de uma comunidade galego-portuguesa nos depoimentos das pessoas visitantes procedentes de Portugal. Esse continuum identitário galego-português (Bello Vázquez 2015b), ou identidade euro-regional transnacional (Pereiro 2016), estaria sustentado na identificação de vários elementos compartilhados: a geografia, a paisagem, a idiossincrasia, os costumes e as tradições, a história e, em menor medida, a língua (também em Rodríguez Prado 2015b e em Fernández Rodríguez, del Río e Rodríguez Prado 2016).

\section{Síntese conclusiva}

Parece-nos oportuno anotar ainda as seguintes questões, transversais ao conjunto de resultados do projeto analisado:

1. No que diz respeito às ferramentas construídas e/ou utilizadas na primeira fase do projeto apresentada neste contributo, verificamos a utilidade do trabalho por questionário e por entrevista para o levantamento e posterior análise de informações sobre os discursos e as práticas sustentadas polas populações analisadas. $\mathrm{Pa}$ ra além de atender as necessidades da equipa nesse sentido, o desenho e elaboração da base de dados destinada ao censo, catalogação e categorização de materiais documentais permitiu também transferir essa tecnologia e compartilhar essa experiência com outras equipas no âmbito internacional. Ainda nesse sentido, constatamos a produtividade do uso quer de técnicas e métodos referenciados no PLN quer de programas informáticos de vário tipo destinados ao manuseamento e exploração de corpora volumosos e variados como os utilizados no projeto em foco.

2. Em relação com a equipa do projeto, verificamos a tendência para uma transdisciplinaridade real, constatável tanto nos processos conduzentes à elaboração das ferramentas referidas acima (base de dados, inquéritos e entrevistas), como na participação conjunta em eventos científicos de áreas diversas (sociologia, antropologia, letras, turismo, gastronomia, ...) por integrantes da equipa procedentes de culturas acadêmicas e áreas de investigação também diferentes (economia, ecologia, linguística, literatura, ...). Da mesma maneira, toda a vez que isso não supõe por enquanto uma prática habitual no campo académico, parece-nos importante constatar a atenção prestada polo pessoal investigador que participa no projeto à reflexão e a (auto)reflexividade sobre a relação com o objeto de estudo, sobre a posição da própria equipa e sobre os impactos (internos e externos) do trabalho investigador por ela desenvolvido.

3. Verificamos a relevância das informações levantadas e do conhecimento gerado nesta primeira fase do projeto tanto para a transferência como para a planificação das comunidades envolvidas, nomeadamente em termos de sustentabilidade local de práticas e consumos, de análise de impactos produzidos por atividades como o turismo e de elaboração de políticas públicas, neste último caso toda a vez que o conhecimento revela-se imprescindível para uma gobernança realmente informada e participada.

4. Já quanto aos resultados das pesquisas realizadas no seio do projeto, destaca a confirmação de hipóteses de partida, quer quanto aos parâmetros da cultura quer, sobretudo, quanto aos seguintes elementos presentes nas três macronarrativas que explicam a imagem e os discursos atuais sobre Compostela (a funcionar não raro como metonímia da Galiza) e os seus Caminhos: 1) a identificação do Caminho com uma certa ideia (religiosa, cristã) da Europa, onde Compostela é a meta do trajeto e a rota o lugar onde se produz o processo de conversão e superação católicas (ideia construída pola Igreja católica); 2) a visão patrimonial e medievalizante (não isenta de religiosidade) construída nos discursos de organizações internacionais como a UNESCO; e 3) o forte impacto dos discursos (perpassados de espiritualidade, autoconhecimento e estética new age, ...) de Paulo Coelho nas práticas, nos consumos e nos discursos dos visitantes (sobretudo) brasileiros, onde a realização do Caminho acaba por ser uma prova de procura, transformação e felicidade individual, assente esta numa conceção religioso-místico-espiritual da vida.

5. Ainda neste sentido, é palpável a ressemantização dos espaços da cidade e a invisibilização das comunidades e culturas locais. Os trabalhos apontam para a construção de uma imagem de Compostela como um santuário com a Catedral no seu centro e da cidade velha como um cenário para práticas e consumos espirituais ou esotéricos próprios de espaços turistificados onde a comunidade local não interage.

6. Parece oportuno referir ainda nesta síntese de resultados que nas informações extraídas dos visitantes portugueses também se verifica a existência de um continuum identitário 
galego-português, uma comunidade galego-portuguesa sustentada sobretudo na comum geografia e paisagem, na idiossincrasia similar das suas gentes, na história compartilhada e, bem que em menor medida, na familiaridade da língua de aquém e além Minho.

7. Por último, entre os aspetos ainda em desenvolvimento e que serão abordados nos trabalhos da fase do projeto iniciada em 2018 destacamos apenas a necessidade de implementação das fases 2 e 3 do Catalogador, destinadas à catalogação automática e à geolocalização de materiais documentais, respetivamente; assim como o interesse na documentação de processos e na traçabilidade e replicabilidade da investigação. Neste sentido, a fixação do processo de elaboração dos inquéritos e a sua publicação, assim como a disponibilização pública e gratuita das bases de dados (de corpus documental e de inquéritos) geradas no projeto, por colocarmos apenas os elementos que julgamos de mais relevância, poderão ser abordados proximamente em função dos recursos disponíveis.

\section{Referências bibliográficas}

Andereck, Kathleen L. e Gyan P. Nyaupane (2011): "Exploring the Nature of Tourism and Quality of Life Perceptions among Residents", Journal of Travel Research 50/3, pp. 248-260.

Ateljevic, Irena; Annette Pritchard e Nigel Morgan (eds.) (2007): The Critical Turn in Tourism Studies. Innovative Research Methodologies. Amsterdão: Elsevier.

Bello Vázquez, Raquel (2014): "Discursos Religiosos, Recriação Histórica e 'Cultura do Caminho' nos Caminhos de Santiago", Mosaico. Revista de História 7/1, pp. 35-44.

(2015a): "Measuring Identity: Indicators for Comparison of Real and Fictional Discourses on a Cultural Heritage City", em G. R. Ricci (ed.), Travel, Tourism, and Identity. New York: Routledge, pp. $127-142$.

(2015b): "A existência de uma comunidade luso-galega como elemento afetivo nas visitas a Santiago de Compostela procedentes de Portugal. Primeiros resultados através de inquéritos quantitativo-qualitativos", em Elias J. Torres Feijó, Raquel Bello Vázquez, Roberto Samartim e Manuel Brito-Semedo (eds.), Estudos da AIL em Teoria e Metodologia. Relacionamento nas Lusofonias II. Santiago de Compostela / Coimbra: Associação Internacional de Lusitanistas / AIL Editora, pp. 87-96.

(2015c): "Homologias entre discursos literários, práticas sociais e consumos culturais. Um estudo de caso com peregrinos brasileiros a Compostela". Comunicação inédita. Colóquio O Brasil perante os desafios do século XXI: literatura, cultura, sociedade (Budapeste, 12-13 de outubro de 2015). Budapeste: Universidade de Budapeste, Associação Internacional de Lusitanistas.

(2016): "Consumos Literários, Práticas Culturais e Habitus: O Paradoxo de Paulo Coelho e o Ca-

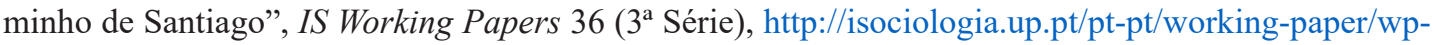
36-consumos-liter\% $\%$ C3\%A1rios-pr\% $\%$ C3\%A1ticas-culturais-e-habitus-o-paradoxo-de-paulo-coelho-e-o [consulta: 26/06/2019].

Carral, Emilio; Xosé C. Carreira; Breogán Martínez Vila e Elias J. Torres Feijó (2016): “A inter-relação entre gastronomia, cultura, turismo e comércio local em Santiago de Compostela. Primeiros resultados", em M. L. Tibério et al. (orgs.), Livro de Atas do III Seminário 'Alimentos e manifestações culturais'e II Simpósio Internacional 'Alimentação e Cultura: Tradição e Inovação na Produção e Consumo de Alimentos'. [Vila Real]: Universidade de Trás-os-Montes e Alto Douro, pp. 263-280.

Church, Kenneth W. e Patrick Hanks (1990): "Word association norms, mutual information, and lexicography”, Computational Linguistics 16/1, pp. 22-29.

Colombo, Jonathan (2011): "Community and Community Development: Conceptual Clarifications", Innovation/Innovación/Inovação. RICEC 3/1, em Two essays about community engagement. Tese de Mestrado HEC, Montreal, pp. 13-43, http://biblos.hec.ca/biblio/memoires/2012NO30.PDF.

Conde, Fernando (2009): Análisis sociológico del sistema de discursos. Cuadernos Metodológicos. Vol. 43. Madrid: Centro de Investigaciones Sociológicas (CIS).

Fernández Rodríguez, María Luisa (2012): A Cripta do Apóstolo e Santiago de Compostela contada aos nenos: Imaxinarios contemporáneos do Camiño de Santiago e Compostela en dous discursos literarios infanto-xuvenís galegos. Trabalho Final de Mestrado inédito. Santiago de Compostela: Universidade de Santiago de Compostela.

(2015): "Peregrinación metodológico-textual por los caminos de Santiago y la Compostela literarias: las etapas de catalogación, digitalización y text mining y el corpus como meta". Comunicação 
inédita. International Conference of Digital Literary Studies (14-15 de maio de 2015). Coimbra: Universidade de Coimbra.

(2016a): Discursos sobre Santiago de Compostela y el/los Camino(s) de Santiago en la novela española actual (2010) a través de técnicas analíticas digitales: Posibilidades y valor del conocimiento generado. Tese de doutoramento inédita. Santiago de Compostela: Universidade de Santiago de Compostela.

(2016b): "Análisis cultural de discursos ficcionales sobre Santiago de Compostela y el Camino de Santiago: exploración de métodos estadístico-computacionales para la categorización empírica del contenido léxico-semántico de corpus voluminosos", em A. Gabriela Macedo e C. Manuel de Sousa, Outros mapas: linguagem, migração, diáspora. Braga: Universidade do Minho, Centro de Estudos Humanísticos, pp. 277-298.

Fernández Rodríguez, María Luisa; Marisa del Río e Felisa Rodríguez Prado (2016): "Dous produtos e um destino: The way (2010), O diário de um mago (1987) e a imagem da Galiza como destino turístico", IS Working Papers 32 (3 $3^{a}$ Série), http://isociologia.up.pt/pt-pt/working-paper/wp-32-dos-produtos-e-umdestino-way-2010-o-di\%C3\%A1rio-de-um-mago-1987-e-imagem-da-galiza [consulta: 26/06/2019].

Fernández Rodríguez, María Luisa e Roberto Samartim (2015a): "Métodos não-supervisionados de clustering para a análise de textos literários: a seleção de materiais sobre grandes volumes de corpus". Comunicação inédita. Congresso de Humanidades Digitais em Portugal (8-9 de outubro de 2015). Lisboa: Universidade Nova de Lisboa.

(2015b): "Análise estatística do discurso para a exploração e classificação de textos literários: extração de conteúdos do romance jacobeu em espanhol de 2010". Comunicação inédita. II Simposium Internacional EDISo 2015 (18-20 de junio de 2015). Coimbra: Universidade de Coimbra.

(2016): "Impacto dos discursos literários nas práticas e consumos culturais ligados aos Caminhos de Santiago: de Paulo Coelho aos souvenirs de cavaleiros templários", IS Working Papers 37 (3ª́rie), http://isociologia.up.pt/pt-pt/working-paper/wp-37-impacto-dos-discursos-liter\%C3\%A1rios-naspr\%C3\%A1 ticas-e-consumos-culturais-ligados-aos [consulta: 26/06/2019].

Frost, Warick (2006): "Braveheart ed Ned Kelly: historic films, heritage tourism and destination image", Tourism Management 27/2, pp. 247-254, https://doi.org/10.1016/j.tourman.2004.09.006.

Garcia, Marcos e Pablo Gamallo (2015): "Yet Another Suite of Multilingual NLP Tools", em J.-L. SierraRodríguez, J.-P. Leal e A. Simões (eds.), Languages, Applications and Technologies. Communications in Computer and Information Science. Springer International Publishing Switzerland, pp. 65-75, https:// doi.org/10.1007/978-3-319-27653-3.

Guinovart, Xavier G. e Alberto Simões (2009): "Parallel corpus-based bilingual terminology extraction", em Marie-Claude L'Homme e Sylvie Szulman (eds.), TIA-2009. Conference on Terminology and Artificial Intelligence. CEUR. Working Proceedings, vol. 578, http://ceur-ws.org/Vol-578/.

Iriarte, Álvaro; Pablo Gamallo e Alberto Simões (2018): “Estratégias Lexicométricas para Detetar Especificidades Textuais", Linguamática 10/1, pp. 19-26, https://doi.org/10.21814/1m.10.1.263.

Lage, Xesús; Antón Losada e Marta Gómez (2012): "La política cultural en la comunidad autónoma gallega: de la dependencia a la autonomía”, RIPS. Revista de investigaciones políticas y sociológicas 11/3, pp. 115-148.

Light, Duncan (2007): "Dracula tourism in Romania Cultural identity and the state", Annals of Tourism Research 34/3, pp. 746-765.

López Pena, Zósimo (2017): "Indicadores culturais relacionados co constructo identitario do Camiño de Santiago comunicados a través de contidos audiovisuais”. Comunicação inédita. I Colóquio Internacional Galabra. Tecendo Rede. Sentidos, aplicações e cooperação na investigação em ciências humanas e sociais (9 e 10 de janeiro de 2017). Braga: Universidade do Minho, Rede Galabra.

Martínez Vila, Breogán Antón (2014): Discursos, imagens e práticas culturais: análise e repercussão dos consumos gastronômicos em Santiago de Compostela de brasileiros, portugueses e espanhóis. Trabalho Fim de Mestrado inédito. Santiago de Compostela: Universidade de Santiago de Compostela.

Pazos-Justo, Carlos (2017): "Turismo, imagem e comunidade. Reflexões a partir do caso de Santiago de Compostela", em F. Groba e P. Dono (orgs.), Galegos no Minho. 20 anos do Centro de Estudos Galegos. Braga: Universidade do Minho, Centros de Estudos Galegos, pp. 79-92, http://hdl.handle. net/1822/49490. 
Pazos-Justo, Carlos; Marisa del Río e Roberto Samartim (2018): "Reinventio e unanimidade. Impacto das políticas culturais e turísticas na Comunidade local de Santiago de Compostela", SÉMATA, Ciencias Sociais e Humanidades 30, pp. 233-256.

Pereiro Pérez, Xerardo (2016): “Turistas portugueses na Galiza: Imagens e relatos pós-experienciais”, em J. M. Trillo Santamaría e I. Pires (coords.), Las fronteras en la investigación peninsular: temáticas y enfoques contemporáneos. Santiago de Compostela: Universidade de Santiago de Compostela, pp. 161-181.

Pichel Iglesias, Irene (2015): Best-sellers, Galicia y la imposibilidad de la identidad: un enfoque cuantitativo. La narrativa histórica actual como miscelánea didáctica. Trabalho Fim de Mestrado inédito. Santiago de Compostela: Universidade de Santiago de Compostela.

Rodríguez Prado, Felisa. (2015a): "Santiago de Compostela em produtos literários brasileiros: lugar (d) escrito ou apenas meta dos Caminhos?". Comunicação inédita. Vème Colloque luso-hispano-francés. Géographie, Langue et Textes Littéraires (23-24 de abril de 2015). Porto: Universidade do Porto.

- (2015b): "Textos ficcionais e práticas culturais de portugueses com relação a Santiago de Compostela: contrastes e homologías", em E. J. Torres Feijó, R. Bello Vázquez, R. Samartim e M. BritoSemedo (eds.), Estudos da AIL em Teoria e metodologia. Relacionamento nas Lusofonias I. Santiago de Compostela / Coimbra: AIL Editora, pp. 143-158.

Ruiz, Esteban e Macarena Hernández (2007): "Identity and community Reflections on the development of mining heritage tourism in Southern Spain”, Tourism Management 28, pp. 677-687.

Samartim, Roberto (2015): "Bases de dados para o estudo da cultura: apresentação do catalogador e possibilidades de abordagem sobre o corpus documental do Projeto Caminho de Santiago", em E. J. Torres Feijó, R. Bello Vázquez, R. Samartim e M. Brito-Semedo (eds.), Estudos da AIL em Teoria e metodologia. Relacionamento nas Lusofonias II. Santiago de Compostela / Coimbra: AIL Editora, pp. 115-126.

(2016): "Tecnologia para os estudos na cultura: Caminhos e ferramentas para a catalogação, a análise léxico-semântica e o mapeamento de materiais", em A. Gabriela Macedo e C. Manuel de Sousa, Outros mapas: linguagem, migração, diáspora. Braga: Universidade do Minho, Centro de Estudos Humanísticos, pp. 261-275.

(2019): "Sustentabilidade investigadora e resiliência académica. Esboço para uma autoanálise". Comunicação inédita. III Encontro Internacional da Rede Galabra, Responsabilidade e Investigação em Ciências da Cultura (24-25 de janeiro de 2019). Corunha / Santiago de Compostela: Universidade da Corunha / Universidade de Santiago de Compostela.

Samartim, Roberto e Miguel Rettenmaier (2015): "Desenho de ferramentas digitais para a catalogação e a exploração de informação e de documentação: os casos do Projeto Caminho de Santiago e do Acervo Literário de Josué Guimarães". Comunicação inédita. International Conference of Digital Literary Studies (14-15 de maio de 2015). Coimbra: Universidade de Coimbra.

Squire, Shelagh J. (1994): "The cultural values of literary tourism", Annals of Tourism Research 21/1, pp. 103-120.

Torres Feijó, Elias J. (2011): "Discursos contemporâneos e práticas culturais dominantes sobre Santiago e o Camiño: a invisbilidade da cultura como hipótese", em A. Apolinário Lourenço e O. Manuel Silvestre (coords.), Literatura, espaços, cartografia. Coimbra: Centro de Literatura Portuguesa, pp. 391-449.

(2012): "Interesses culturais e âmbitos receptivos em dous romances sobre o Caminho de Santiago: Frechas de Ouro e O Enigma de Compostela", Romance Notes 52/2, pp. 135-149.

(2013): "Sustainable identity. Tourism, as opportunity or menace. Proposal of some indicators". International Critical Tourism Studies Conference, https://www.academia.edu/4178554/Sustainable identity_Tourism_as_opportunity_or_menace_Proposal_of_some_indicators [consulta: 26/06/2019].

(2014): "Autor, texto e espaço geo-cultural mediatizado: processos de ressemantização, banalização e misturação de géneros em produtos sobre o Caminho de Santiago", em S. Quinteiro e R. Baleiro (eds.). Lit\&Tour: Essays on Literature and Tourism. Lisboa: Húmus, pp. 291-310.

(2015a): "Comunidades, coesão, sustentabilidade e fator visitante: ferramentas conceituais e parâmetros para a análise: o caso de Santiago de Compostela", em C. Almeida Marques (org.), Planeamento Cultural Urbano em Áreas Metropolitanas - Revitalização do Espaço Pós-Suburbano. Lisboa: CAPP-ISCSP-ULisboa \& CIAUD-FA-U (cap. 7: “Arte e Cultura, Factores de Identidade Urbana e Desenvolvimento da Economia"), pp. 121-123.

(2015b): "Tourism and local community: unperceived and imperceptible risks and socially responsible research (SRR). First stages to work SRR with traders". Comunicação inédita. VI International Critical Tourism Studies Conference. Faculty of Tourism and Hospitality Management (26-30 
de julho de 2015) Opatija, Croácia, https://www.academia.edu/13124788/Tourism_and_local_community_unperceived_and_imperceptible_risks_and_socially_responsible_research_SRR_._First_stages_ to_work_SRR_with traders [consulta: 26/06/2019].

(2015c): "Identity Sustainability, Identity Affectivity, and the Ithaca Traveler: Conceptual Tools for Measuring and Modeling Tourism as an Opportunity", em G. R. Ricci (ed.), Travel, Tourism and Identity, Culture \& Civilization 7, pp. 143-162.

Torres Feijó, Elias J. e María Luisa Fernández Rodríguez (2014): "Literary discourse and local repercussions: Paulo Coelho and the cultural practice in Santiago de Compostela, capital of Galicia". Comunicação inédita. 14th conference of the International Society for the Empirical Study of Literature and Media (21-25 de julho de 2014). Turim: Università degli Studi di Torino.

Villarino Pardo, Carmen (2015): "Produtos literarios e práticas culturais sobre Santiago de Compostela: proposta de análise contrastiva dos corpus (romances e inquéritos)", em E. J. Torres Feijó, R. Bello Vázquez, R. Samartim e M. Brito-Semedo (eds.), Estudos da AIL em Teoria e metodologia. Relacionamento nas Lusofonias I. Santiago de Compostela / Coimbra: AIL Editora, pp. 89-104.

Xie, Philip Feifan; Halifu Osumare e Awad Ibrahim (2007): "Gazing the hood: Hip Hop as tourism attraction", Tourism Management 28/2, pp. 452-460. 